\title{
FALTA INJUSTIFICADA DEL PERSONAL DE ENFERMERÍA A SU JORNADA LABORAL: UN ENFOQUE EXPLICATIVO.
}

\author{
UNJUSTIFIED ABSENCE OF NURSES TO THEIR WORKING DAY: AN EXPLICATIVE APPROACH
}

JULIO ALFREDO GARCÍA PUGA.*

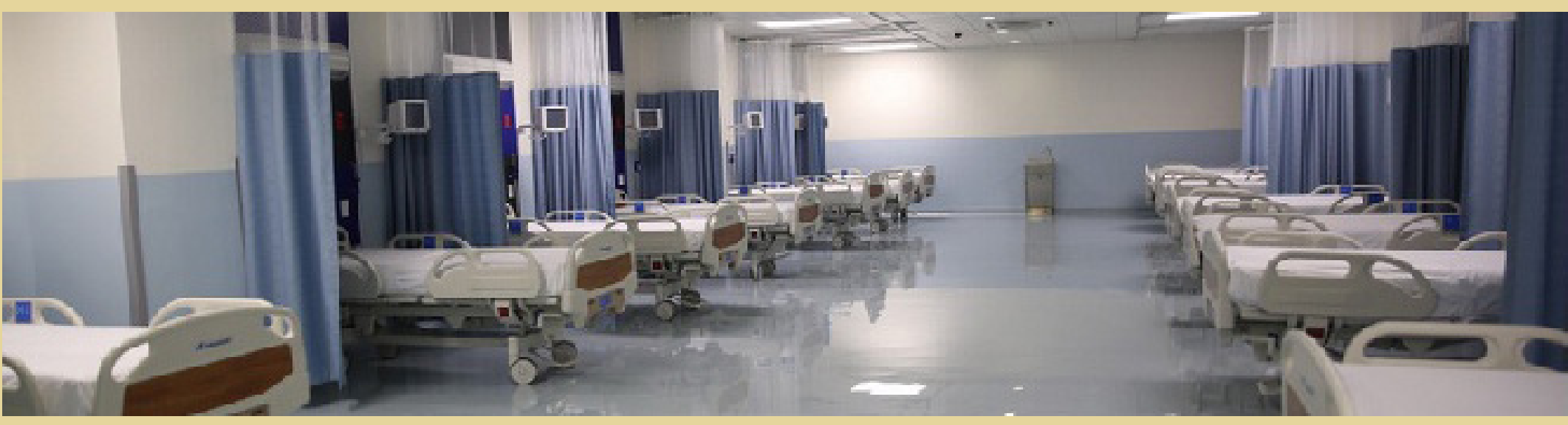

\begin{abstract}
This essay is a reflection on the phenomenon of unjustified absence of nurses to their workday at a public hospital in Sonora, Mexico, which is a second level hospital dependent of the Health Secretary. Although the phenomenon has been studied from different approaches and has raised the multidimensional nature of the problem, in this essay is approached from the perspective of social capital of nurses and the logic of social practices, for it is used as a theoretical approach to the notion social capital and Pierre Bourdieu practical reason, all articulated with the structural working conditions of the hospital institution. Based on the exposed by Ives Morieux is done the proposal to articulate six institutional rules in nursing practice as a coadjuvant to prevent unexcused absence of nurses. Such as preliminary conclusions considers that nurses are products of their living conditions and employment status therefore instead of blaming individuals the structure could be responsible for labor unexcused absence.
\end{abstract}

Key words: labor Absenteeism, unjustified absence, absence

\section{RESUMEN:}

E n este ensayo se propone una reflexión sobre el fenómeno de la inasistencia no justificada del personal de enfermería a su jornada laboral en un público del estado de Sonora, el cual es un hospital de segundo nivel dependiente de la Secretaria de Salud. Si bien el fenómeno ha sido estudiado desde diferentes enfoques y se ha planteado el carácter multidimensional del problema en este ensayo se aborda desde la perspectiva de capital social de las enfermeras y la lógica de la practicas sociales, para ello se utiliza como enfoque teórico la noción de capital social y la razón práctica de Pierre Bourdieu, todo ello articulado con las condiciones laborales estructurales de la institución hospitalaria. A partir de lo expuesto por Ives Morieux se hace la propuesta de articular las seis reglas en práctica institucional de enfermería como coadyuvante para evitar la inasistencia no justificada de las enfermeras. Como conclusiones preliminares se considera que las enfermeras son producto de sus condiciones de vida y de su situación laboral, Por ello en lugar de culpabilizar a los individuos la estructura podría ser la responsable de la inasistencia injustificada laboral.

$\left.{ }^{*}\right)$ Doctor en Ciencias . Docente de tiempo complete. Departamento de Enfermería. Universidad de Sonora. Email: juliopuga1@hotmail.com
Palabras clave: Absentismo laboral, ausencia injustificada, absentismo. 

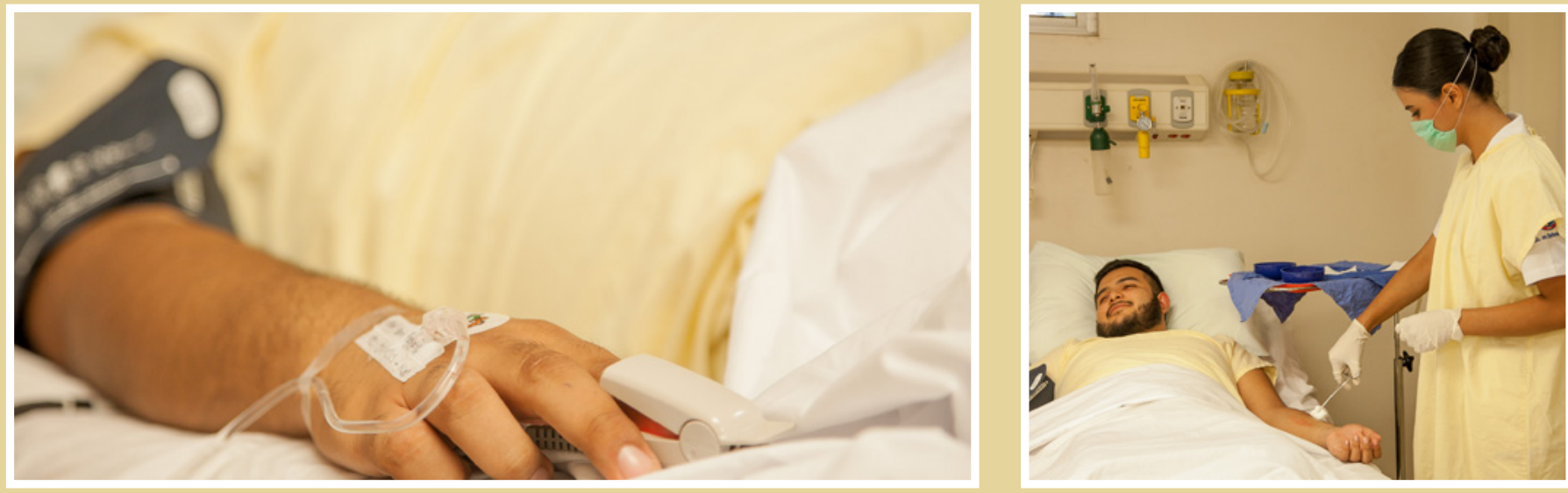

\section{EL PROBLEMA DEL AUSENTISMO LABORAL NO PROGRAMADO}

Antes de abordar directamente el fenómeno del ausentismo no programado o falta no justificada conviene establecer que esta se refiere a cualquier inasistencia no programada del trabajador, llámense permiso económico, incapacidad médica, incapacidad materna, pago de guardia o falta, lo que es coincidente con lo establecido por la Organización Internacional del Trabajo donde se establece como la no asistencia al trabajo por parte de un empleado que se pensaba iba a asistir.

Este ausentismo es costoso tanto para el trabador como para la empresa y suele ser reflejo de la satisfacción o insatisfacción del empleado en la empresa. Puesto que empleados que se sienten comprometidos con la institución suelen presentar menores índices de ausentismo no programado que aquellos que se perciben insatisfechos con su trabajo ${ }^{(1)}$.

En el caso del personal sanitario y especialmente para enfermería esta situación se vuelve sensible por las diversas funciones que realizan en el sistema de salud; quienes se han constituido como el pilar de las instituciones de salud, por ello, es necesario que el propio personal de enfermería conozca sus funciones y categorías en las cuales se desenvuelve lo que determinará la concor- dancia entre funciones y categoría laboral, y por ende mayor satisfacción laboral (2).

Ante ello el primer esfuerzo importante por establecer las categorías y funciones inherentes a ellas se estableció en la Norma Oficial Mexicana NOM-019-SSA3-2013 ${ }^{(3)}$, para la práctica de enfermería en el Sistema Nacional de Salud donde se considera como enfermera a la persona que ha concluido sus estudios de nivel superior en el área de la enfermería, en alguna institución perteneciente al Sistema Educativo Nacional y se le ha expedido cédula de ejercicio con efectos de patente por la autoridad educativa competente, para ejercer profesionalmente la enfermería; existiendo diversos grados académicos [tabla 1]. En el sistema de salud mexicano existen-laboran enfermeras con diferentes grados de formación que van de auxiliares de enfermería a enfermeras con posgrado, pasando por enfermeras con cursos pos técnicos en enfermería y licenciadas en enfermería. 
TABLE 1. ClasifiCACión DEL PERSONAL DE ENFERMERÍA

\begin{tabular}{|c|c|}
\hline Grado & Definición \\
\hline enfermera(o) general & $\begin{array}{l}\text { A la persona que obtuvo título de licenciatura en enfermería ex- } \\
\text { pedido por alguna institución de educación del tipo superior, per- } \\
\text { teneciente al Sistema Educativo Nacional y le ha sido expedida } \\
\text { por la autoridad educativa } \\
\text { competente la cédula profesional correspondiente }\end{array}$ \\
\hline enfermera(o) especialista & $\begin{array}{l}\text { A la persona que, además de reunir los requisitos de licenciado } \\
\text { en enfermería, obtuvo el diploma de especialización en términos } \\
\text { de las disposiciones jurídicas aplicables y le ha sido expedida por } \\
\text { la autoridad educativa competente la cédula profesional de espe- } \\
\text { cialización correspondiente a un área específica de competencia }\end{array}$ \\
\hline enfermera(o) con maestría: & $\begin{array}{l}\text { A la persona que, además de reunir los requisitos de licenciatura } \\
\text { en enfermería, acredita el grado de maestría, en términos de las } \\
\text { disposiciones jurídicas aplicables y le ha sido expedida por la au- } \\
\text { toridad educativa } \\
\text { competente la cédula profesional de maestría correspondiente }\end{array}$ \\
\hline enfermera(o) con doctorado & $\begin{array}{l}\text { A la persona que, además de reunir los requisitos del grado de } \\
\text { maestría, acredita el grado de doctorado, en términos de las dis- } \\
\text { posiciones jurídicas aplicables y le ha sido expedida por la auto- } \\
\text { ridad educativa } \\
\text { competente la cédula profesional de doctorado correspondiente }\end{array}$ \\
\hline profesional técnico de enfermería & $\begin{array}{l}\text { A la persona que obtuvo el título o un diploma de capacitación } \\
\text { expedidos por alguna institución perteneciente al Sistema Educa- } \\
\text { tivo Nacional. }\end{array}$ \\
\hline técnico especializado en enfermería: & $\begin{array}{l}\text { A la persona que, además de reunir los requisitos de estudios de } \\
\text { nivel medio superior, obtuvo un diploma que señala el área espe- } \\
\text { cífica de competencia expedido por alguna institución de educa- } \\
\text { ción, perteneciente al Sistema Educativo Nacional }\end{array}$ \\
\hline $\begin{array}{l}\text { personal no profesional de enfer- } \\
\text { mería }\end{array}$ & $\begin{array}{l}\text { A la persona que no ha concluido su preparación académica en } \\
\text { el área de la enfermería en alguna institución educativa perte- } \\
\text { neciente al Sistema Educativo Nacional o habiéndola concluido } \\
\text { no ha obtenido el documento correspondiente que demuestre } \\
\text { tal circunstancia, para que la autoridad educativa competente la } \\
\text { autorice para ejercer dicha actividad. En esta clasificación se en- } \\
\text { cuentran la auxiliar de enfermería, los estudiantes y pasantes de } \\
\text { enfermería }\end{array}$ \\
\hline auxiliar de enfermería: & $\begin{array}{l}\text { A la persona que obtuvo constancia que acredite la conclusión } \\
\text { del curso de auxiliar de enfermería, de una duración mínima de } \\
\text { un año expedido por alguna institución perteneciente al Sistema } \\
\text { Educativo Nacional }\end{array}$ \\
\hline
\end{tabular}

Fuente: NORMA Oficial Mexicana NOM-019-SSA3-2013, Para la práctica de enfermería en el Sistema Nacional de Salud. 
Esta situación genera en las enfermeras diversas formas de visualizar su responsabilidad ante el trabajo y principalmente hacia los usuarios, por lo que se espera que aquellas personas con mayor nivel académico sean más responsables que aquellas con menor grado de formación.

El impacto hacia el propio empleado por una falta no programada se traduce en su economía personal, en nuestro caso y esto dependerá del nivel de ingreso del trabajador los descuentos por falta suelen rondar alrededor de los 1, 000 pesos mexicanos por día. ${ }^{1}$ Por otro lado para la institución hospitalaria se generan costos administrativos por el manejo de las ausencias y lo más importante, se viola el derecho a la salud que cada mexicano tiene y que se establece en el Artículo IV constitucional que dice: Toda persona tiene derecho a la protección de la salud.

Esta violación o incumplimiento del mandato constitucional -en el caso de la inasistencia injustificada por enfermería- se da una vez que se ausenta una enfermera y los pacientes que deberían ser atendidos por ella se deben de re-distribuir entre el personal que se encuentre laborando, lo que impacta directamente en forma negativa en la salud de los pacientes: así a mayor número de pacientes atendidos por una enfermera mayor es el riesgo de error en la atención, complicaciones biomédicas e insatisfacción por los usuarios; para la enfermera la sobrecarga de trabajo le genera insatisfacción, cansancio y frustración por no poder realizar su trabajo con la calidad necesaria. ${ }^{(4)}$

1

a- El término enfermeras se refiere tanto a varones como mujeres que ejercen esta profesión.

b- El sueldo de una enfermera depende del nivel salarial con la que esta cuente, y se integra por el sueldo base más prestaciones y una compensación económica mensual al salario del personal federal.

De acuerdo al reporte generado por el servicio de jefatura de enfermería del Hospital Infantil del Estado de Sonora las faltas injustificadas son importantes para el hospital y se presentan variaciones en diferentes momentos del año e incluso de los días. Ejemplo de ello fue en el mes de diciembre en el cual se presentaron poco más de 500 faltas entre los diversos turnos, lo que en promedio representaron 16.5 faltas injustificadas por día. Situación alarmante.2

2- Reporte de incidencias de jefatura de enfermería HIES, 2014.

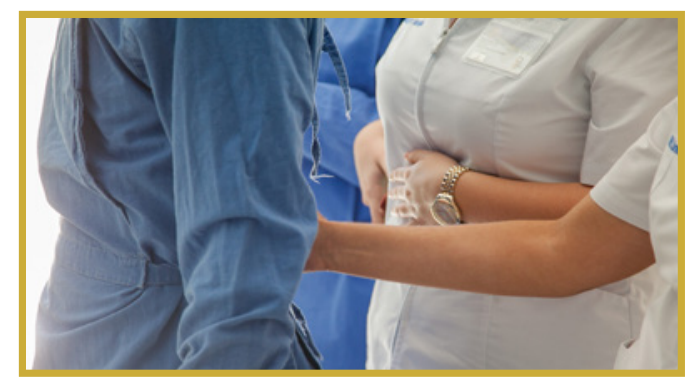

En relación a la estructura del sistema de salud mexicano en donde desarrollan su práctica las enfermeras- por su financiamiento existen instituciones de salud de carácter público quienes reciben los recursos de forma tripartita (estado, patrón y empleado) o bipartita (gobierno federal y estatal) y un sistema de atención privado que se sustenta en base al pago directo de los usuarios o de instituciones de seguros médicos. ${ }^{(5)}$

Esta división condiciona que existan relaciones laborales diferenciadas, así en aquellas de carácter pública se han establecido garantías sindicales que favorecen la inasistencia del empleado. En el caso de las instituciones hospitalarias dependientes del gobierno estatal de Sonora los trabajadores cuentan dos sindicados importantes por la cantidad de sus agremiados: Sindicato Único de los Trabajadores al Servicio de los poderes del Estado de Sonora (SUTSPES), Sindicato Nacional de los Trabajadores de la Secretaria de Salud sección 43 (SNTSA).

Para comprender el fenómeno de la inasistencia es necesario no solo conocer la estructura donde se desarrolla la práctica de enfermería, es imperante además contar con una base conceptual que nos ayude a entender el entramado fenómeno, para ello el capital social y las razones prácticas nos ayudaran a su comprensión. Desde la perspectiva de Bourdieu el capital social son las relaciones sociales con las que cuentan los individuos y en las cuales acceden a ventajas producto de esas relaciones, mientras que las razones prácticas se sustentan en la lógica de los individuos las cuales pueden ser incongruentes con las ideas o representaciones sociales de una práctica profesional de enfermería. ${ }^{(6,7)}$ 
En el caso de las enfermeras, y al igual que otros sujetos sociales, ellas (os) son producto de un proceso que inicia con su formación académica, que posteriormente se articulará con la práctica hospitalaria, y en función del tiempo se ira estructurando y reestructurando hasta formar un habitus de enfermería. O en palabras de Bourdieu como un sistemas de disposiciones duraderas y transferibles, estructuras estructuradas predispuestas a funcionar como estructuras, es decir, como principios generadores y organizadores de prácticas y de representaciones ${ }^{(7)}$.

\section{LAS CAUSAS DE INASISTENCIA}

\section{La estructura}

En el contrato colectivo del SUTSPES ${ }^{(8)}$ en su artículo 47 fracción XII establece: Queda estrictamente prohibido al trabajador faltar sin causa justificada. Este artículo es claro en la obligación del trabajador de no faltar de forma injustificada a sus obligaciones laborales, sin embargo, se presenta una ambigüedad y contradicción con el articulo al interior del mismo contracto, ya que si bien por un lado existe la prohibición por otro, establece el derecho de faltar hasta por tres días en un periodo de 30 días sin perjuicio para el trabador, salvo la percepción económica.

Además en la institución no existe un sistema de competencia que estimule al trabajador para no presentar inasistencias. Si un trabajador presenta hasta tres faltas cada mes sus derechos permanecen inalterados, o sea puede gozar de los mismos días económicos, pases de salida, vacaciones, oportunidades de cambio de jornada laboral etc., que el personal que no falta a su trabajo.

Además, la sobrecarga de trabajo no le genera al personal que tiende a los pacientes ninguna ganancia económica o reconocimiento por las autoridades.

Por otra parte los usuarios no tienen la capacidad de decidir quién es la enfermera que desean los atiendan, por lo cual están supeditados a la oferta institucional. Esto puede impactar en la competencia profesional que se genera entre las enfermeras, así si una enfermera es solicitada para su atención debería ser estimulada social y económicamente por su trabajo.

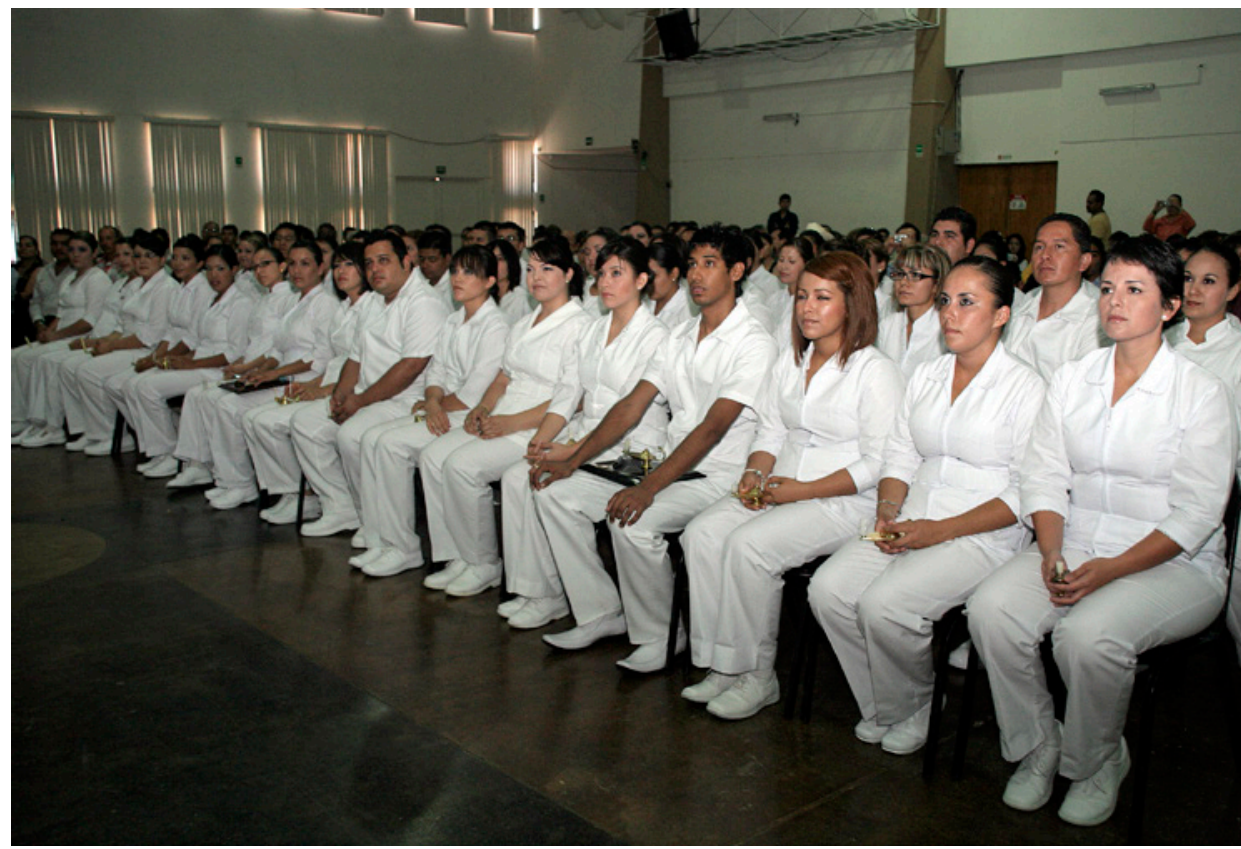

\section{El capital social y la lógica de la práctica}

El capital social condiciona una forma de ver la realidad y actuar en concordancia a dicha visión, por lo cual las personas con mayor capital cultural y económico tienden a tener mayor responsabilidad laboral, muestra de ello en el caso de enfermería se observa por el nivel académico que poseen. En el trabajo de Ledesma encontró que el personal con nivel de licenciatura en enfermería el porcentaje de inasistencias es menor que la enfermera general-técnico en enfermería [ $10.9 \%$ vs $48.76 \%$ ]. ${ }^{(10)}$

Esta situación aparentemente refleja que las licenciadas en enfermería al contar con mayor instrucción académica y mayor relación con otras disciplinas durante el periodo de formación universitaria generan una mayor responsabilidad laboral y ética hacia los usuarios de salud.

Además al cuestionar a algunas enfermeras del porqué de la falta se encuentran una amplia diversidad de respuestas que van desde porque "tenía flojera", "no pasa nada", "nomas", "porque tuve mucho trabajo la jornada anterior". Lo importante en las respuestas anteriores es la muestra de una falta de integración del trabajador en la institución labora. Esto es, existe un desencanto ante el trabajo y hacia los pacientes. 


\section{Algunas sugerencias}

Debido a la complejidad del fenómeno y bajo el supuesto que los individuos somos producto de las condiciones de vida, y laborales, considero que la propuesta en la ponencia de Ives Morieux "simplificar el trabajo" se pueden adaptar las seis reglas en el caso de enfermería y con ellas desde la estructura hospitalaria reforzar la noción de responsabilidad del personal de enfermería hacia los pacientes.

La propuesta de Morieux se basa en el cambio paradigmático de la gestión administrativa, al pasar desde una visión centrada en la estructura a la comprensión y retroalimentación entre el personal y la estructura. ${ }^{111}$

Los seis puntos propuestos se pueden adaptar a nuestro medio a través de:

1. Conocer que hace la gente: es importante que el personal directivo administrativo tome conciencia de las funciones que realizan las enfermeras en relación a su categoría laboral con el propósito de establecer los límites de su competencia, además deben de darse a conocer dichas funciones entre el personal.

2. Reforzar los integradores: es imperante que el personal directivo se capaciten con el propósito de gestionar los recursos humanos basados en una práctica científica y ello proponer a la vez reforzadores entre el personal de enfermería que atiende a los usuarios.

3. Incrementar la cantidad de poder: Conocimiento es poder. Esta expresión es vital en la disciplina de enfermería, ya que una práctica fundamentada en los resultados de las investigaciones les proporcionan un marco de trabajo que favorecerán la integración con la institución y los usuarios de la atención de enfermería.

4. Extender la sombra del futuro: usualmente la práctica de enfermería se desconecta entre las diferentes áreas hospitalarias, por ello la práctica se vuelve irreflexiva. Al extender la sombra de las acciones se toma en cuenta la integración entre los diversos servicios, evitando prácticas desconec- tadas entre sí. Ejemplo de ello se puede ver en el traslado del paciente entre los servicios, si la enfermera que atiende en un primer momento al usuario brinda la atención integral cuando el paciente sea atendido en un servicio receptor se evitaran duplicidad de funciones, pérdida de tiempo y una mejor atención para el usuario.

5. Incrementar la productividad: es imperante que las enfermeras tomen conciencia de la "productividad" de sus acciones. Los empleadores pueden hacer explícito como se puede medir tal productividad a través de diversos indicadores.

6. Recompensar a aquellos que cooperan: es sumamente importante este punto, ya que el personal que cumple con su obligación laboral debe ser recompensando, facilitándoles los procesos administrativos o con estímulos económicos. Mientras que para aquellos que faltan reiteradamente deben de ser no recompensados.

\section{CONCLUSIONES}

A través de los expuesto anteriormente podemos concluir que el personal de enfermería es producto de las condiciones estructurales los cuales promueven de manera oculta la inasistencia del personal, sin embargo es a partir de la compresión de este fenómeno que se pueden llevar a cabo estrategias que modifiquen en primer término a la estructura y posteriormente a los individuos. La propuesta de Morieux puede ser un acercamiento para abatir el ausentismo laboral no justificado.

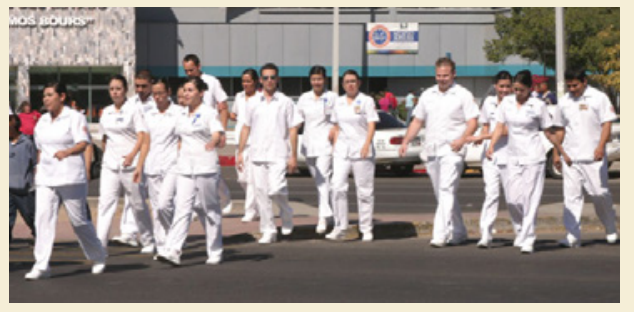

SONUS - DEPARTAMENTO DE ENFERMERÍA 


\section{REFERENCIAS}

1. Sánchez GD. El absentismo laboral en España. Del ordenamiento jurídico a la realidad empresarial. Tesis doctoral. 2013. Universidad Rey Juan Carlos. España.

2. Secretaria de Salud. Día de la enfermera 2014. Mensaje de la Secretaria de Salud, Mercedes Juan. [internet]. 2014. [acceso 30 de junio de 2014]. Disponible en:

http://www.salud.gob.mx/unidades/ $\mathrm{cie} / \mathrm{cms}$ _cpe/?Id_URL=notas\&Id_Nota $=264 \&$ anio $=2014$.

3. Diario Oficial de la Federación. Norma Oficial Mexicana NOM019-SSA3-2013, Para la práctica de enfermería en el Sistema Nacional de Salud. [internet]. 2013. [acceso 20 de junio de 2014]. Disponible en: http://www.dof.gob.mx/nota_detalle.php?codigo $=5312523 \& \mathrm{fe}-$ cha $=02 / 09 / 2013 \&$ print $=$ true .

4. Instituto de Investigaciones Jurídicas. Constitución política de los estados Unidos Mexicanos título Primero articulo IV. [internet]. 2009. [acceso 20 de junio de 2015]. Disponible en: http:// info4.juridicas.unam.mx/ijure/fed/9/5. htm?s.

5. Gómez DO, Sesma S, Becerril M,
Knaul F, Arreola H. y Frenk J. Sistema de Salud de México. Salud Pública de México. 2011; 53 S2: S220-232.

6. Bourdieu P. Razones prácticas: Sobre la teoría de la acción. 2da. Ed. España: Anagrama. 1997.

7. Bourdieu P. El sentido práctico. Argentina: Siglo XXI Editores. 2007.

8. López F, Alcanzar FM y Romero FP. Una revisión del concepto y evolución del capital social. [internet]. 2007. Disponible en dialnet.unirioja.es/servlet/ articulo? codigo $=2233299$.

9. Gobierno del Estado de Sonora, Servicios de Salud de Sonora, SUTSPES. Contrato colectivo de Trabajo. 2013.

10. Ledesma SS. Causas de ausentismo del personal de enfermería en un hospital general. 2008. Tesis de maestría. Universidad Michoacana de San Nicolás de Hidalgo.

11. Ives Morieux. Seis reglas para simplificar el trabajo conforme se vuelve más complejo. [internet]. 2013. [acceso 30 de junio de 2015]. Disponible en: http://www.ted.com/talks/yves_morieux_as_work_gets_more_complex_6_ rules_to_simplify?language $=\mathrm{es} \# \mathrm{t}-2311$.

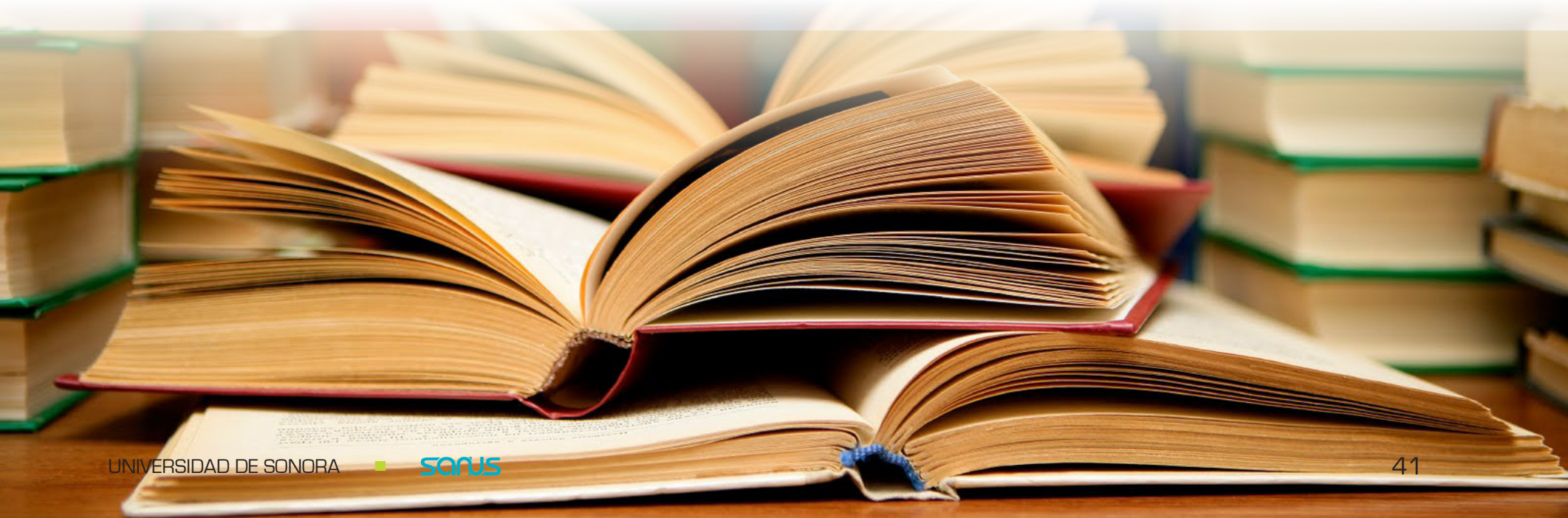

